\title{
An Update on Pediatric Keratoprosthesis
}

\author{
Mohammad H Bawany ${ }^{1}$, Faraaz Khan ${ }^{2}$ and James Aquavella ${ }^{2 *}$ \\ ${ }^{1}$ University of Rochester School of medicine and dentistry, USA \\ ${ }^{2}$ Flaum Eye Institute, University of Rochester medical center, USA
}

*Corresponding author: James Aquavella, MD. Flaum Eye Institute, University of Rochester Medical Center.

Received Date: December 28, 2018

Published Date: January 11, 2019

\section{Opinion}

As we see ourselves one year closer to the year 2020, we ask ourselves if we are closer to accomplishing the World Health Organization's "Vision 2020," a project aimed at eliminating the main causes of avoidable blindness by the year 2020 [1]. A top priority of this ambitious initiative is the prevention of childhood blindness. Blindness in children is complex; it affects, in addition to eyes, a child's neurologic development. Further, childhood blindness requires resources from and collaboration between medical, community, and educational services to nurture the growing child. Providing sight to a child who may otherwise go blind is thus more than just about restoring vision [2].

Early blindness has been shown to have far-reaching complications for the affected child. Studies have shown that blind infants have impeded motor development, including delayed head control and neck control, and disturbed calibration of limbal proprioception. It has been hypothesized that vision is necessary for normal motor development by providing feedback to a child's vestibular and proprioceptive systems [3]. While studies have not shown any significant IQ differences in blind children compared to their seeing counterparts, they have indicated that children with congenital visual impairment have discrepancies in language presentation. Though they may develop normal grammar, vocabulary, and other components that allow a person to communicate, their pragmatic language skills may be hindered, as these develop with visual cues from interactions in various social contexts [2].

Ensuring vision is thus vital to a child's development, and upwards of $40 \%$ of causes of childhood blindness are preventable or treatable [2]. Conditions that lead to corneal opacification can fall under these potentially treatable causes of amblyopia, childhood blindness. These conditions can include congenital corneal opacities as seen in Peter's anomalies and acquired scarring of the cornea as seen in congenital glaucoma, infectious keratitis, or Stevens-Johnson syndrome. In these cases, the time a child is not seeing must be minimized to prevent the development of amblyopia. A number of procedures, including keratoprosthesis (KPro), the implantation of an artificial cornea, have been made available to offer corneal transplantation to those affected by these conditions $[4,5]$.

Keratoprosthesis was once considered a surgery only for adults and was used as a last resort that was often riddled with complications. Due to modifications of the device itself, alterations to implantation techniques, and improvements to post-operative regimens, the use of KPro has dramatically increased and has come with improved outcomes. In the last few decades, KPro has been offered as a primary corneal surgery in a number of conditions and has also become available for pediatric populations. Alternatives to KPro in infants include penetrating keratoplasty (PKP) and optical iridectomy. Each technique comes with its own set of challenges: PKP can be complicated by the development of astigmatism, which, if it progresses undetected, can cause dense ambylopia. PKP also relies on an allograft and thus comes with a risk of graft rejection and its associated complications. Further, data on PKP graft survival has been less than compelling, with 1-year survival rates less than $30 \%$ in some studies [6]. Optical iridectomy is limited in that it can only be used for opacities that do not involve the entire corneal surface, and only in cases where the eye is otherwise diseasefree. It is not the best option for infants with congenital glaucoma, cataracts, and vitreous and retinal disease in addition to a corneal opacity, as may often be the case [7].

Keratoprosthesis uses a clear, immunologically inert material with a central optic that allows for image transmission to replace the cornea. It does not use an allograft and thus comes with no risk of rejection. It also provides a clear visual axis from the first day after surgery and thus comes with no risk of deprivation amblyopia. The surgery does require lifelong commitment to ophthalmic drops, occasional visits to replace contact lenses placed over the prosthesis, and high rates of retroprosthetic membrane formation. 
The surgery also requires lens removal and replacement to prevent cataract formation and often requires Ahmed valve placement to prevent the development of glaucoma [6].

The last decade has seen advances in the keratoprosthesis design, operative procedure, and post-operative care that have led to better outcomes and fewer procedure-associated complications in adults. Data from pediatric cases is still limited and continues to be collected and compiled to further cater the procedure to the youngest of patients. From adult data, modifications were made to the previously solid backplate used in the device. Fenestrations were added to allow aqueous humor circulation to the ocular surface. This modification led to improved nutrition of the ocular surface and resulted in decreased rates of corneal melting (keratolysis). A further modification included adding a bandage contact lens to overlay the prosthesis to further enhance its integrity. This has led to further decreases in rates of corneal melting. Additionally, post-operative antibiotics and steroids have been used to decrease infections and inflammatory responses that could lead to corneal melt as well. Despite improved long-term outcomes, sterile corneal melting is still a significant problem and accounts for a majority of device failures in adults and children. Corneal melt is a significant problem because it can lead to KPro extrusion and may be followed by vision loss secondary to aqueous leakage, endophthalmitis, choroidal hemorrhage, or retinal detachment [8].

Understanding the risk factors for corneal melt is essential for developing effective prophylactic and treatment approaches, especially in pediatric KPro cases. There is a causal relation between retroprosthetic membrane (RPM) formation, a common complication seen after KPro in infants, and corneal melt. Removal of RPM from prosthesis backplate holes may not always be practical or feasible to prevent corneal melt, but future approaches to reduce RPM formation are being examined. For instance, using a titanium backplate that is more RPM resistant instead of the current standard polymethyl methacrylate, or using an oversized backplate that hinders RPM formation may be options available in future devices. Further, stressed epithelium produces matrix metalloproteinases (MMP), which may lead to corneal degeneration seen in keratolysis. Strategies to inhibit MMP are being further investigated to protect and stabilize the epithelial layer after KPro. Medications such as ACEI and statins, known to decrease MMP activity, are being studied to determine their efficacy in preventing keratolysis [8]. In adults, local and also systemic infliximab therapy have shown a role in preventing corneal melt after KPro surgery [9,10]. In 2017 Fung and colleagues presented Canadian results with the pediatric KPro with 11 children in their series. Of note, retroprosthestic membranes occurred in more than $80 \%$ of children in this series and sterile keratolysis occurred in $45 \%$ [11].

Pediatric KPro has been offered at the Flaum Eye Institute at the University of Rochester Medical Center since 2004 and has yielded promising results for certain patients. It must be noted that successful KPro implantation and follow-up requires a multidisciplinary team of eye specialists. The corneal surgeon, a pediatric glaucoma specialist, a vitreoretinal specialist, and a pediatric ophthalmologist must all be part of the team to ensure the best possible outcomes. A vitreoretinal specialist, for instance, can perform a pars plana vitrectomy and photocoagulation to address retinal damage after prosthesis insertion. This can decrease the chance of retinal detachment secondary to the invasive prosthesis insertion procedure. A glaucoma specialist can perform appropriate procedures to mitigate glaucoma risks associated with congenital corneal scarring and invasive surgeries [7].

In 2014, a more recent change to the KPro procedure, using a conjunctival flap to completely cover the transplant after implantation, was made by surgeons at the Flaum Eye Institute. Conjunctival flaps have been used in various ocular surface disorders, where they serve as a structural reinforcement for the cornea, and a physical barrier to prevent any direct corneal damage. As it is also well-vascularized tissue, the conjunctival flap can additionally promote healing of a damaged corneal surface it overlays. Though a mainstay in the treatment of various ocular surface disorders such as corneal ulcers and burns, the use of a conjunctival flap in KPro has yet to gain complete traction, and its effects on outcomes of the KPro procedure have yet to be fully elucidated.

\section{Acknowledgment}

None.

\section{Conflicts of Interest}

No conflicts of interest.

\section{References}

1. Blindness: Vision (2020) The Global Initiative for the Elimination of Avoidable Blindness.

2. Pring L, Tadic V (2010) The Cognitive and Behavioral Manifestations of Blindness in Children. In: Nass RD and Frank Y (eds.) Cognitive and Behavioral Manifestations of Pediatric Diseases. New York, Oxford University Press, USA.

3. Prechti H, Cioni G, Einspieler C, Bos AF, Ferrari F (2001) Role of vision on early motor development: lessons from the blind. Developmental Medicine \& Child Neurology 43(3): 198-201

4. Aquavella JA (2018) Controversy in the Management of Congenital Cornea Opacification. J0J Ophthal 6(2): 555682.

5. Aquavella JV, Wozniak R (2018) Benefits of Multidisciplinary Team Approach to Infant Keratoprosthesis. Int J Ophthal Vision Res 2(1): 0810.

6. Trief D, Marquezan MC, Rapuano CJ, Prescott CR (2017) Pediatric Corneal Transplants. Curr Opin Ophthalmol 28(5): 477-484

7. Aquavella JV (2011) Study shows value of KPro in Youngest Eyes. Review of Ophthalmology.

8. Bouhout S, Robert MC, Deli S, Harissi-Dagher M (2018) Corneal melt after keratoprosthesis: clinical presentation, management, outcomes and risk factor analysis. Ocular Immunology and Inflammation 26(5): 693-699.

9. Robert MC, Črnej A, Shen LQ, Papaliodis GN, Dana R, et al. (2017) Infliximab after Boston Keratoprosthesis in Stevens-Johnson Syndrome: An Update. Ocul Immunol Inflamm 25(3): 413-417.

10. Wozniak RA, Gonzalez M, Aquavella JV (2016) Keratoprosthesis in Ectodermal Dysplasia. Cornea 35(7): 1026-1028.

11. Fung SSM, Jabbour S, Harissi-Dagher M, Tan RRG, Hamel P, et al. (2018) Visual outcomes and complications of type I Boston keratoprosthesis in children. Ophthalmology 125(2): 153-160. 